\title{
FACTOR VII AND HEPARIN IN THROMBOSIS
}

\author{
BY \\ L. POLLER* \\ From the Department of Pathology, Withington Hospital, Manchester
}

(RECEIVED FOR PUBLICATION AUGUST 25, 1958)

Factor VII is the term used by Koller, Loeliger, and Duckert (1951) for the accelerator complex of prothrombin conversion present in both plasma and serum which is markedly reduced by treatment with drugs of the dicoumarin group.

It has been shown by Poller (1954 and 1956a) that there is a significant reduction in the anticoagulant action of heparin on the plasma clotting times of patients with recent thrombo-embolic disease. It has also been shown by Poller (1957) that there was a significant increase in plasma factor VII activity in such patients at the same stage of their illness.

The aim of the present investigation was to determine whether there was any association between the increase in factor VII activity and the "heparin resistance" of these cases. The problem is of particular interest because both factor VII and heparin are of established importance in maintaining the normal balance of coagulation.

\section{Method of Study}

To assess the effect of a factor VII preparation on heparin plasma clotting times, increasing volumes of the factor VII preparation were added to plasma clotting mixtures containing a standard amount of the heparin anticoagulant. In a second series of tubes serum rich in factor VFI was substituted for the factor VII preparation, and in a third normal saline was used as a control.

\section{Reagents}

Platelet-rich Plasma.-Fresh oxalated plasma was obtained by spinning venous blood collected without undue venostasis into a siliconed syringe and diluted 1 in 10 with $1 \%$ potassium oxalate.

Heparin.-Standard solution of heparin 10 units $/ \mathrm{ml}$. (Evans).

\section{Calcium Chloride (0.25\%).}

Stored Serum.-This was obtained from normal venous blood allowed to clot at $37^{\circ} \mathrm{C}$., separated, and the serum stored at room temperature for 24 hours. The factor VII activity of the resulting serum in

\footnotetext{
*Present address: Department of Clinical Pathology, Manchester Royal Infirmary.
}

restoring the prothrombin defect in "dindevan" plasma was approximately twice that of the original plasma, volume for volume.

\section{Factor VII Preparation}

This was prepared from normal stored serum by adsorption and elution using the method described by Biggs and Macfarlane (1953). The resulting factor VII activity was many times that of the original serum, volume for volume.

\section{Procedure}

Three rows of six tubes were assembled in a $37^{\circ} \mathrm{C}$. water-bath. To each tube $0.5 \mathrm{ml}$. of plasma and $0.1 \mathrm{ml}$. (1 unit) of heparin were added. To the first row of tubes volumes of physiological saline were added from $0.1 \mathrm{ml}$. in the second to $0.5 \mathrm{ml}$. in the sixth.

To the second row similar volumes of normal stored serum were added, and to the third row the same increasing volumes of the factor VII solution.

To each tube in the first row $0.5 \mathrm{ml}$. of $0.25 \%$ calcium chloride was added at staggered 15-second intervals, and the clotting time noted. The procedure was continued at 15 -second intervals through all three rows of tubes. (The clotting times were performed simultaneously to reduce the effect of storage in vitro on the results.)

The experiment was performed three times and the results expressed as an average for the three investigations.

\section{Results}

The results were similar on all three occasions, and the mean values are given in Fig. 1. The clotting time is markedly shortened by the addition of the factor VII solution, and with volumes of $0.3 \mathrm{ml}$. and more the anticoagulant effect of the heparin appears to have been completely neutralized. The results in tubes 4,5 , and 6 are similar to those obtained in a series of normal plasma clotting times using the same technique but without the addition of heparin and factor VII.

Serum rich in factor VII activity has a similar but less marked antagonism to heparin. 


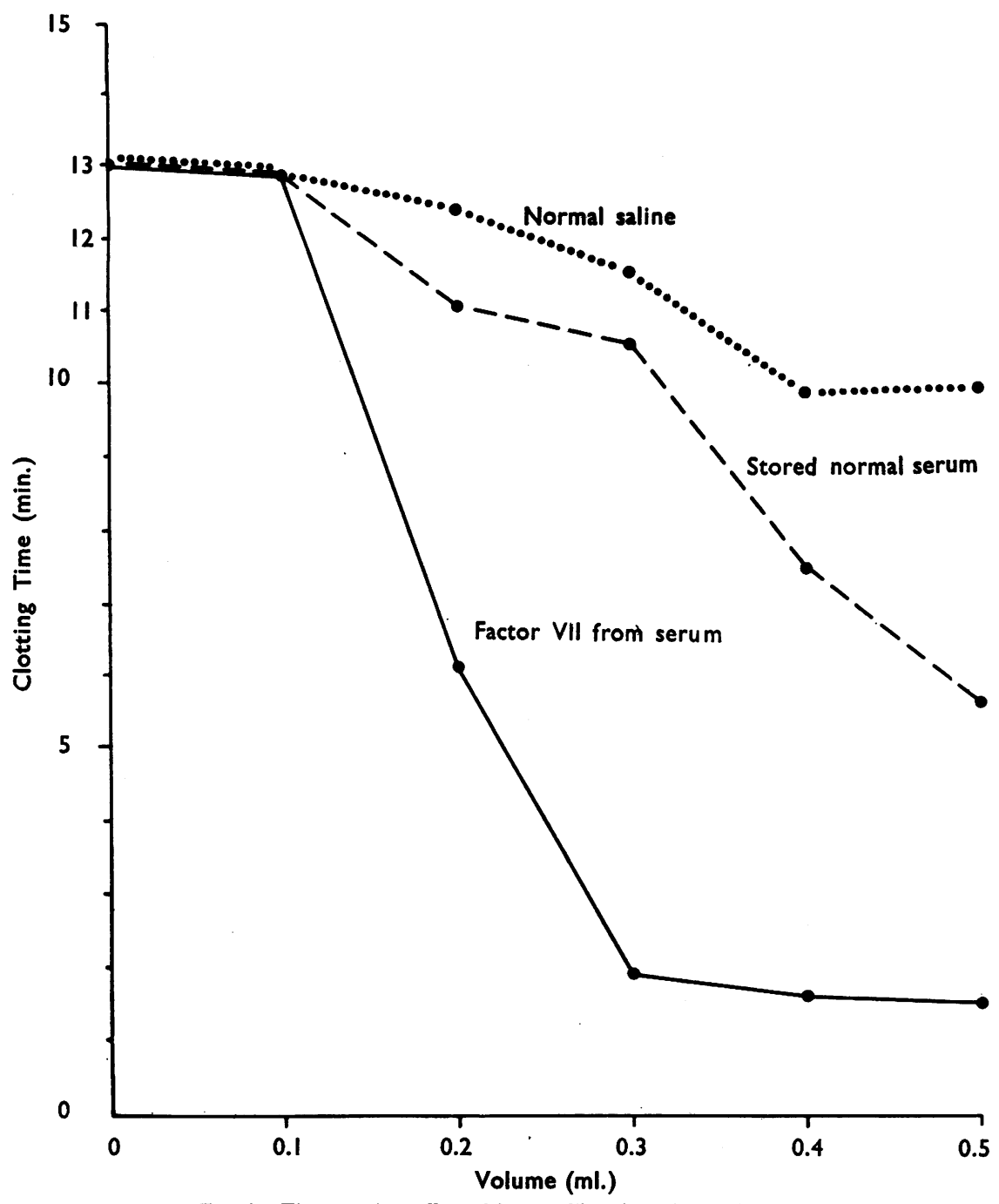

FIG. 1.-The coagulant effect of factor VII on heparin clotting time.

The slight effect produced by saline may have been due to simple dilution of the calcium chloride to an optimum strength for coagulation.

Effect on Thrombin Generation. - Further confirmation of the marked antagonism between a serum factor VII preparation and heparin was obtained from the rate of thrombin generation in plasma clotting in the presence of heparin. The results are given in Fig. 2. Line A shows the slow rate of thrombin generation in heparin plasma clotting.times. (The results here are the mean of three investigations.) In lines $\mathbf{B}, \mathrm{C}, \mathrm{D}$, thrombin generation in three subjects is shown, using an identical technique except for the addition of 0.5 $\mathrm{ml}$. of the factor VII solution to the heparin plasma clotting time. The anticoagulant action of heparin appears to have been completely neutralized by the factor VII solution, the pattern resembling thrombin generation in simple plasma clotting without heparin.

\section{Discuscion}

It may be seen from the above investigations that the factor VII-rich extracts prepared by adsorption and elution of serum possessed a marked antagonistic action to heparin in plasma clotting and in thrombin generation.

The antiheparin activity may be due to one of 0 three other serum factors adsorbed and eluted with factor VII. Van Creveld and Paulssen (1951 


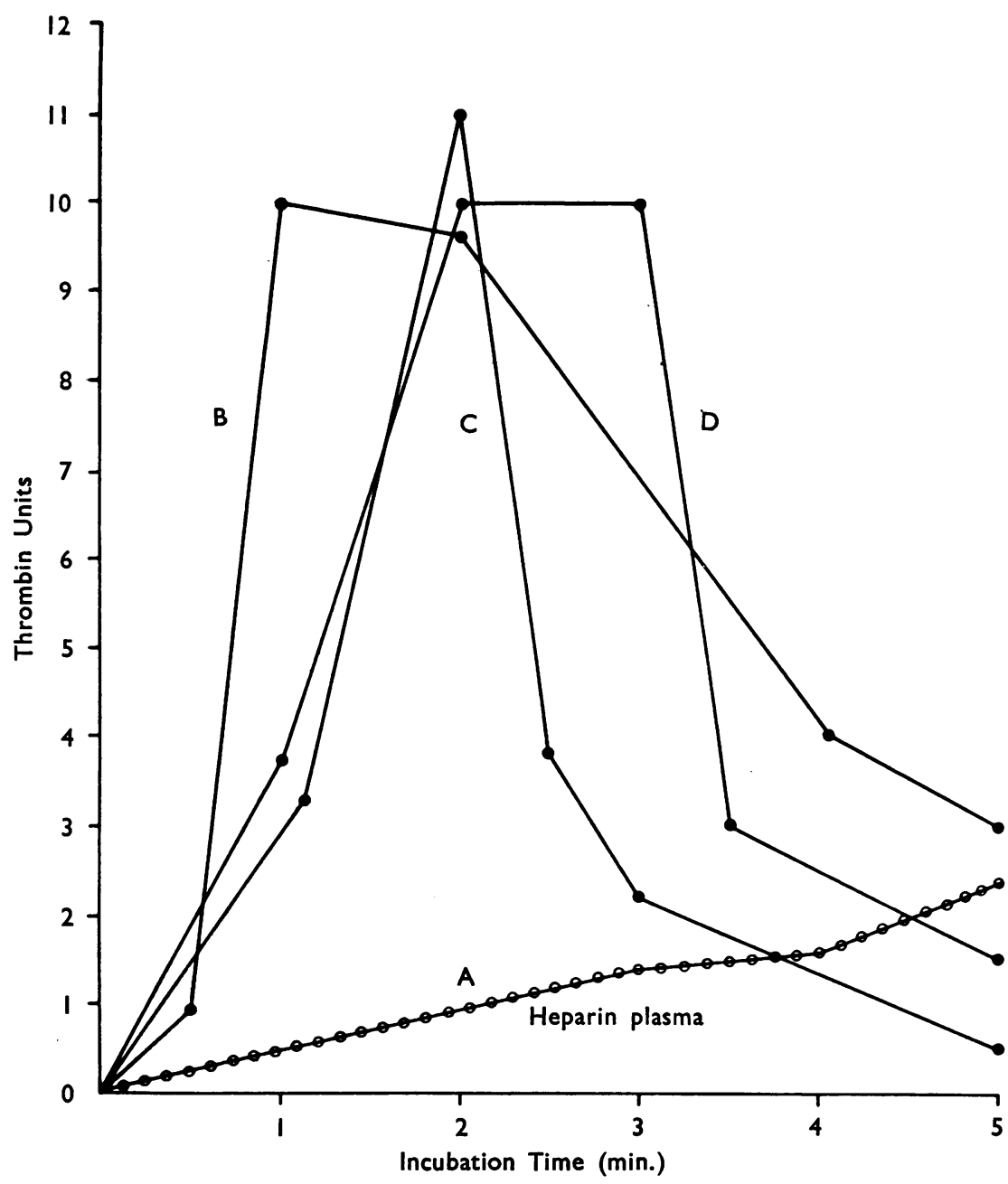

FIG. 2. -The coagulant effect of factor VII preparation $(0.5 \mathrm{ml}$.) on heparin plasma clotting time in three normal adults $(B, C, D)$.

and 1952) described an antiheparin platelet factor. Serum extracts are also known to contain Christmas factor and possibly factor $X$ (Duckert, Flückiger and Koller, 1954) which may have been responsible.

The effects of a factor VII preparation prepared from recalcified platelet-deficient plasma collected into siliconed glassware were found to be equally marked. It thus appears unlikely that the effect is due to the antiheparin factor of platelets.

The antiheparin activity of sera from eight severe cases of Christmas disease was significant using the heparin plasma clotting technique. The effect was less than in a normal group, but the results were not significantly different. Christmas factor therefore does not appear to be the important serum heparin antagonist. Active antiheparin factor VII preparations were made from Christmas serum.

Factor $\mathbf{X}$ is unstable at room temperature and is markedly decreased in liver disease (Duckert et al., 1954). Serum was obtained from cases of parenchymatous liver disease and was stored at room temperature for 48 hours, and showed impaired activity in thromboplastin generation. The resulting serum extract, however, showed marked antiheparin activity in plasma clotting. It seems very unlikely, therefore, that the effect could be due to factor $X$ adsorbed and eluted with factor VII. 


\section{Conclusions}

The demonstration of marked antagonism between factor VII and heparin provides a link between two previous observations in cases of thrombosis. The first was that the anticoagulant action of heparin is less marked in the presence of recent thrombosis, a fact established by De Takats by injection in vivo (1943a and b) and by work in vitro by Waugh and Ruddick (1944a and b), by Rosenthal and Weaver (1952), and by Poller $(1954,1955,1956)$. The second observation made by Poller (1957) was that in a group of patients suffering from recent thrombo-embolic disorders there was significantly increased plasma factor VII activity.

The exact relationship of these findings to abnormal intravascular clotting is not clearly defined. There are two possible explanations. The first is that increased factor VII activity may be a causative agent in the production of thrombosis, neutralizing the natural anticoagulant heparin and upsetting the balance of coagulation. This role is suggested by the experimental work of Wessler (1954, 1955), who produced rapid thrombosis in animals by the infusion of serum rich in factor VII activity. Indirect evidence to support this theory is also provided by Koller et al. (1952), by Ciulla and Santoni (1954), and by Alexander, Meyers, Goldstein, Gurewich, and Grinspoon (1954), who found increased factor VII activity during pregnancy which they presumed to be associated with the "hypercoagulability of pregnancy."

The second possible explanation is simpler, namely, that the increased factor VII activity results from serum factors released into the circulation from the thrombus (serum containing more factor VII than plasma). Indirect support for this may be deduced from the fact that the shortest heparin plasma clotting times occurred in major vessel occlusion, e.g., thrombophlebitis group, mean 4.6 minutes with a standard deviation (S.D.) 1.04, whereas the coronary thrombosis group gave a mean of 6.25 minutes (S.D. 2.1) (Poller, 1956a).

Whatever the exact relationship of these changes the demonstration of increased factor VII activity and the role of factor VII in neutralizing the anticoagulant heparin provide a firm basis for the administration of anticoagulant drugs to restore the disturbed balance of coagulation.

\section{Summary}

Serum extracts rich in factor VII activity prepared by adsorption and elution markedly reduce the anticoagulant action of heparin on plasma clotting and thrombin generation. This fact provides a link between the two previous observations made by the author in cases of recent thrombo-embolic disorders, namely, that there is resistance to the anticoagulant action of heparin and increased plasma factor VII activity.

There is indirect evidence to support two possible theories of the relationship to thrombosis. The first possibility is that factor VII plays an important role in the actual causation of thrombosis by its antiheparin action. The second explanation is that increased plasma activity results simply from the absorption into the circulation of factor VII liberated from the thrombus.

The demonstration of the neutralization of natural anticoagulant by factor VII provides a firm basis for the administration of the dicoumarin type drugs in these conditions, of which the main function is to depress the raised level of factor VII activity.

I should like to thank Dr. M. C. G. Israels and Dr. R. G. Macfarlane for advice on this work, and the Department of Haematology, Manchester Royal Infirmary, for batches of Christmas serum.

\section{REFERENCES}

Alexander, B., Meyers, L., Goldstein, R., Gurewich, V., and Grinspoon, L. (1954). J. clin. Invest., 33, 914.

Biggs, R., and Macfarlane, R. G. (1953). Human Blood Coagulation, p. 343. Blackwell Scientific Publications, Oxford.

Ciulla, U., and Santoni, G. (1954). Haematologica, 38, 575. van Creveld, S., and Paulssen, M. (1951). Lancet, 2, 242.

- (1952). Ibid., 1, 23.

De Takats, G. (1943a). J. Amer. med. Ass., 121, 1246. (1943b). Surg. Gynec. Obstet., 77, 31

Duckert, F., Flückiger, P., and Koller, F. (1954). Rev. Hémat., 9, 489.

Koller, F., Loeliger, H., and Duckert, F. (1951). Acta haemat. (Basel), $6,1$.

Poller, L. (1954). Angiology, 5, 21.

- (1955). Acta haemat. (Basel), 14, 372.

—_ (1956a). Clin. Sci., 15, 55.

—_ (1956b). M.D. thesis, University of Manche:ter.

(1957). J. clin. Path., 10, 348.

Rosenthal, R. L., and Weaver, J. C. (1952). Circulation, 6, 257.

Waugh, T. R., and Ruddick, D. W. (1944a). Canad. med. Ass. J., 50, 547.

- (1944b). Ibid., 51, 11.

Wessler, S. (1954). J. clin. Invest., 33, 972. (1955). Ibid., 34, 647. 